\title{
Des sources de photons uniques... ultrabrillantes
}

\author{
Pascale SENELLART ${ }^{1,2}$, Valérian GIESZ ${ }^{1}$ et Loic LANCO ${ }^{1,3}$ \\ 'Laboratoire de Photonique et de Nanostructures, CNRS-LPN, Route de Nozay, F-91460 Marcoussis \\ 2Département de Physique de l'École polytechnique, F-91 128 Palaiseau Cedex \\ ${ }^{3}$ Université Paris Diderot - Paris 7, F-75205 Paris Cedex 13 \\ pascale.senellart@lpn.cnrs.fr
}

Pour qu'un réseau de communication quantique voie le jour, des sources émettant des photons un à un de façon très efficace sont indispensables. Grâce à une nouvelle technique de lithographie, récompensée par la Médaille d'argent du CNRS en 2014, de telles sources ont récemment été fabriquées à partir de boîtes quantiques, véritables atomes artificiels à l'état solide. Cette lithographie, réalisée à température cryogénique, permet de positionner une boîte quantique unique avec une précision nanométrique au centre d'une microcavité optique.

\section{Des sources de lumière quantique peu efficaces}

Un enjeu majeur pour le développement de réseaux optiques quantiques est la fabrication de sources de photons uniques efficaces. Idéalement, une telle source doit émettre, à la demande, un photon et un seul, dans un mode défini du champélectromagnétique ${ }^{1}$. Aujourd'hui, les sources utilisées dans les protocoles de calculs ou communications quantiques optiques utilisent des sources de photons uniques annoncés : deux photons sont générés par conversion de fréquence paramétrique dans un milieu non-linéaire et la détection d'un photon annonce l'autre. Pour ne pas générer top d'impulsions contenant plus d'un photon, ces sources fonctionnent à un flux tel que moins de $5 \%$ des impulsions contiennent effectivement une paire de photons, les autres étant essentiellement vides. De toute évidence, cette situation n'est pas idéale : elle limite dramatiquement le débit de toute communication optique et, dans le domaine plus large du calcul quantique, tons uniques. Les photons sont bien émis dans un mode défini du champ, mais la statistique des photons est poissonienne : même lorsqu'en moyenne les impulsions contiennent moins d'un photon, la probabilité qu'une impulsion en contienne deux n'est pas négligeable. elle limite la complexité des calculs que l'on peut réaliser.

\section{Une piste : les boîtes quantiques semiconductrices...}

Depuis une quinzaine d'années, une autre approche est explorée pour fabriquer de telles sources. Il s'agit d'utiliser des nano-émetteurs, appelés boîtes quantiques semiconductrices. Ce sont des insertions de taille nanométrique d'un matériau semiconducteur dans un autre, constituant un piège très efficace pour les électrons [1]. Les niveaux d'énergies des porteurs dans une telle nanostructure sont discrets, à l'image des niveaux électroniques dans un atome unique. Lorsqu'un grand nombre de porteurs sont créés dans une boîte quantique, celle-ci émet successivement plusieurs photons, dont la longueur d'onde d'émission dépend directement du nombre de porteurs dans la boîte quantique. Ainsi, à une longueur d'onde donnée, un seul photon est émis. Ces objets constituent la source de photons uniques idéale, au sens où pour chaque impulsion d'excitation, la probabilité pour la boîte quantique d'émettre un photon et un seul est proche de $100 \%$ [2].

En revanche, ces objets souffrent du problème commun à toute source de lumière semiconductrice : la difficulté de collecter la lumière émise, majoritairement piégée par réflexion totale interne dans le matériau. En réalité, si la boîte quantique émet bien un photon à chaque excitation, moins de $1 \%$ sort effectivement du composant... Pour résoudre ce problème, il faut contrôler la façon dont la boîte quantique est couplée à la lumière. Comme l'a prédit E. Purcell en 1946, l'émission spontanée n'est pas une propriété de l'émetteur seul mais dépend de son environnement électromagnétique [3]. En plaçant un émetteur ponctuel dans une cavité optique, il est possible d'accroître son couplage avec un certain mode du champ optique et d'accélérer son émission spontanée dans ce mode. Pour cela, il faut toutefois réunir deux conditions: placer la boîte quantique sur un maximum du champ optique confiné et en résonance spectrale avec celui-ci.

\section{... mais comment les contrôler?}

Obtenir ces conditions s'est avéré être un vrai casse-tête. En effet, les boîtes quantiques se forment spontanément du fait de la différence des paramètres cristallins quand l'on fait croître de l'arséniure d'indium (InAs) dans une matrice d'arséniure 
de gallium (GaAs) : ces îlots ont donc des positions totalement aléatoires. De plus, leur taille latérale est de l'ordre de $20-30 \mathrm{~nm}$ pour une épaisseur de seulement quelques $\mathrm{nm}$, soit une dizaine de monocouches atomiques environ. Ainsi, la moindre variation de forme ou composition change significativement la longueur d'onde d'émission. Dans ce contexte, les techniques de fabrication traditionnelles, qui ne disposent pas d'un contrôle de la position spatiale et spectrale des boîtes quantiques, donnent des rendements de fabrication des composants inférieurs à $0.1 \%$.

Un important effort de recherche a été accompli pour résoudre ce problème. Certaines approches se sont attachées à forcer la croissance des boîtes quantiques sur un réseau prédéfini. Bien que prometteuses, ces méthodes n'ont pas encore permis d'obtenir des boîtes quantiques présentant de bonnes qualités optiques. D'autres techniques ont consisté à mesurer par microscopie à force atomique la position de la boîte par rapport à des marques métalliques, puis à dessiner par lithographie électronique une cavité alignée par rapport à ces marques [4]. S'en suivent un grand nombre d'étapes technologiques pour accorder les longueurs d'onde de la cavité et de la boîte quantique. Spectaculaires, ces techniques sont extrêmement exigeantes et n'ont pas été reproduites.
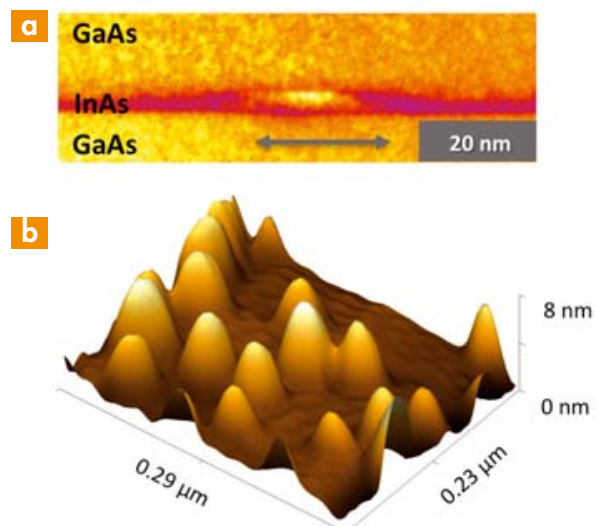

Figure 1. Les boîtes quantiques semiconductrices. (a) Image d'une boîte quantique unique obtenue au microscope électronique à transmission (avec l'aimable autorisation de $G$. Patriarche et $A$. Lemaître, LPN-CNRS). (b) Image obtenue au microscope à force atomique d'un plan de boîte quantique (avec l'aimable autorisation de C. Gomez-Carbonell, LPN-CNRS). (c) Schéma de principe de l'émission de photons uniques : lorsque plusieurs paires électrons-trous sont créées dans la boîte quantique, le système émet plusieurs photons les uns après les autres à des longueurs d'ondes différentes. (d) Spectre d'émission typique d'une boîte quantique à $10 \mathrm{~K}$.
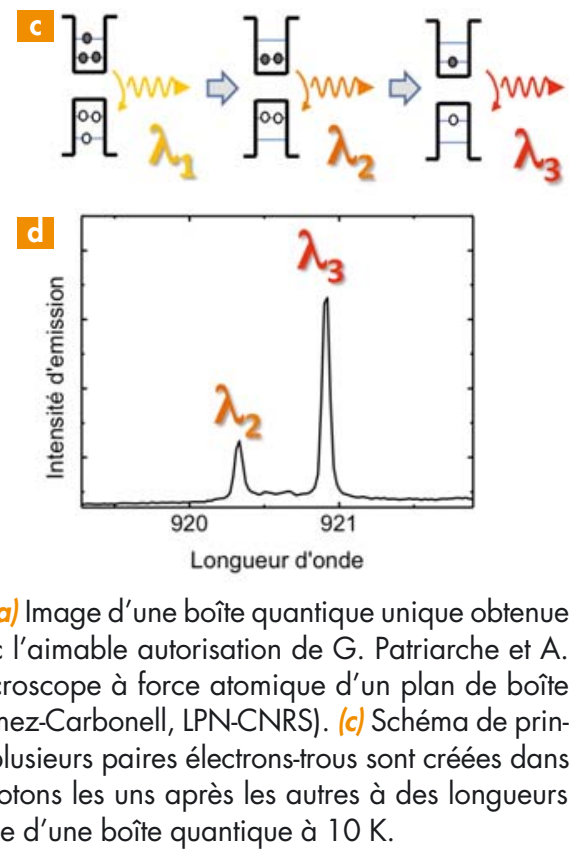

\section{Une solution : la lithographie optique in situ}

Au laboratoire de Photonique et de Nanostructures du CNRS, une approche tout à fait originale a été proposée. Elle consiste à mesurer optiquement la position d'une boîte quantique et à dessiner directement une cavité centrée sur celle-ci .

est déposée sur un échantillon contenant une distribution aléatoire de boîtes un cryostat pour atteindre une température de $10 \mathrm{~K}$, à laquelle les boîtes quantiques émettent des photons uniques de excite l'émission des boîtes quantiques sans affecter la résine photosensible (fi2a-(I)). La tâche d'excitation laser a diamètre limité par la diffraction, de boîte quantique, objet de taille nanométrique, dépend de la densité locale d'excitation. Ainsi, en déplaçant l'émetteur par rapport au faisceau d'excitation, il est possible de mesurer la position de la boîte quantique en maximisant son émission (figure 2a-(II)). De même qu'il est possible de pointer le sommet d'une montagne large de plusieurs kilomètres avec une précision de l'ordre du mètre, il est possible ici de repérer la position de la boîte quantiques. L'échantillon est placé dans grande pureté. Un faisceau laser rouge

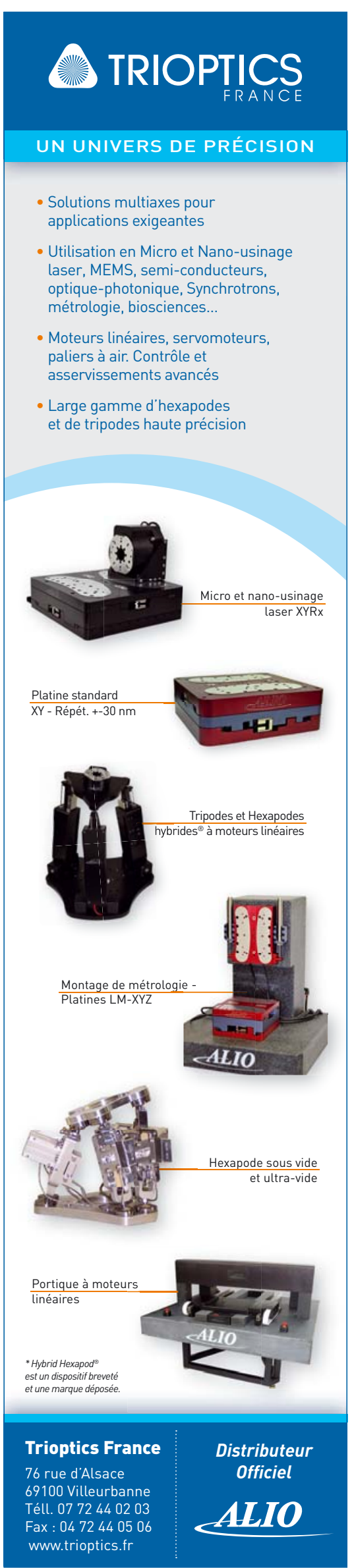


quantique avec une précision de l'ordre de $50 \mathrm{~nm}$. La technique de lithographie in situ consiste ensuite à utiliser un laser vert, parfaitement superposé au laser rouge, pour insoler dans la résine photosensible un motif centré sur l'émetteur, qui définira directement la cavité optique (figure 2a-(III)). Finalement, connaissant la longueur d'onde d'émission de la boîte, la géométrie de la cavité est ajustée pour être accordée à celle-ci précisément. Les étapes de repérage et écriture sont répétées pour fabriquer un grand nombre de composants en une seule étape de lithographie. Les cavités sont ensuite gravées avec les techniques de gravure habituelles des composants optoélectroniques.

La technique de lithographie in situ a été utilisée pour placer de façon contrôlée une boîte quantique dans divers types de cavités. Des cavités micropiliers, obtenues par gravure d'un cylindre de quelques microns de diamètre dans une structure de cavité planaire à miroirs interférentiels, ont servi de première démonstration. Pour augmenter le potentiel de la technique, le CNRS a développé, avec la société attocube, une machine de lithographie in situ qui permet depuis 2010 de réaliser des structures plus complexes : des cavités piliers dites « connectées " pour un contrôle électrique du composant [6], des cavités couplées [7], des cavités hybrides diélectriques-plasmoniques, etc.

\section{Des sources de photons uniques... ultrabrillantes}

En insérant une boîte quantique unique dans une cavité, il est maintenant possible de modifier son émission spontanée à volonté et de fabriquer des sources de photons uniques extrêmement brillantes. Ces deux termes peuvent paraître paradoxaux : en réalité, comme la boîte quantique émet un photon à chaque excitation optique, la brillance décrit ici la probabilité avec laquelle il est possible de le collecter. En l'absence de cavité, cette brillance est limitée à quelques pourcents.

Couplée à une cavité, l'émission de la boîte dans le mode de cavité est accélérée d'un facteur $F_{\mathrm{p}}$ tandis que son taux d'émission dans tout autre mode optique est

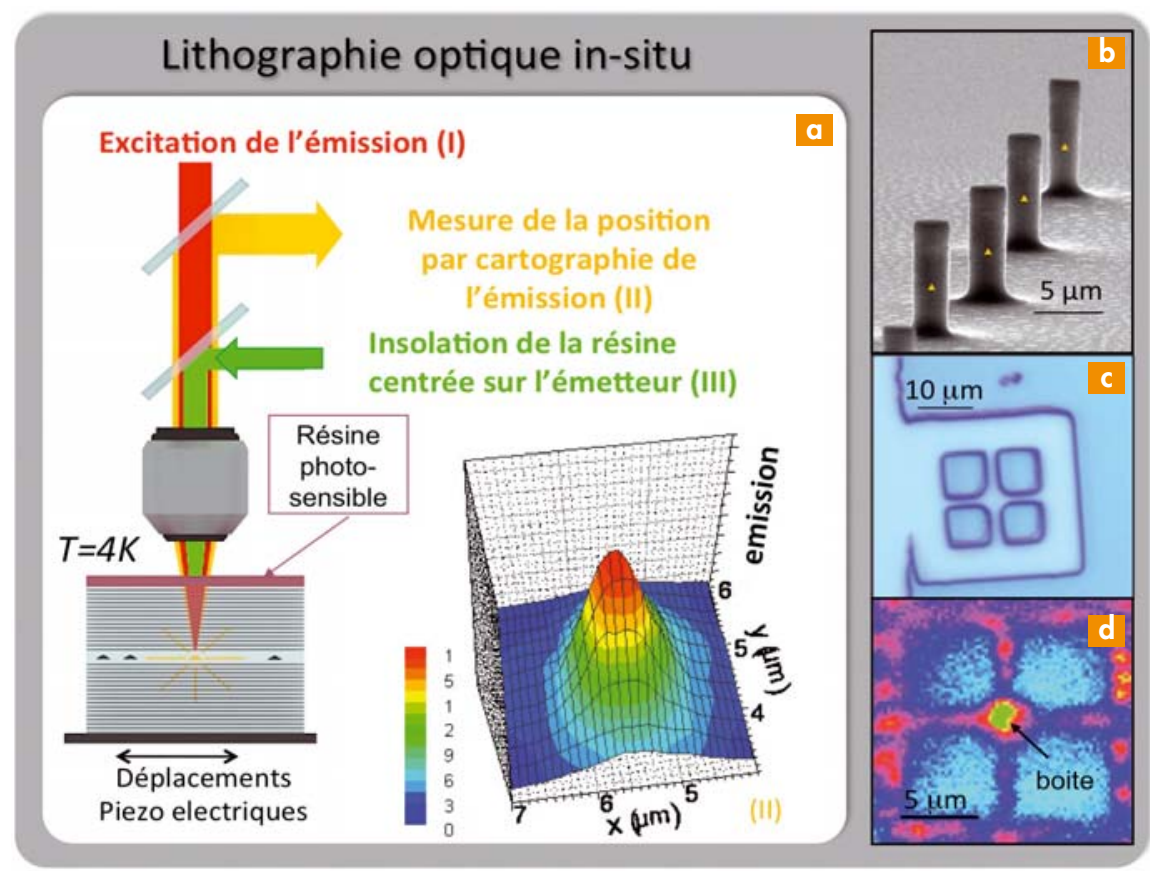

Figure 2. (a) Schéma de principe : un laser rouge permet de mesurer la position des boîtes quantiques en réalisant une cartographie de leur émission (en bas à droite). Un laser vert est utilisé pour dessiner un motif dans la résine photosensible aligné par rapport à la boîte quantique choisie. (b) Image obtenue au microscope électronique à balayage de micropiliers réalisés ainsi : chaque pilier contient en son centre une boîte quantique unique. (c) Image obtenue au microscope optique d'une structure de pilier connecté [6] dessinée dans la résine. (d) Cartographie de l'émission du composant présenté en (c) montrant un fort signal d'émission de la boîte quantique placée au centre du motif.
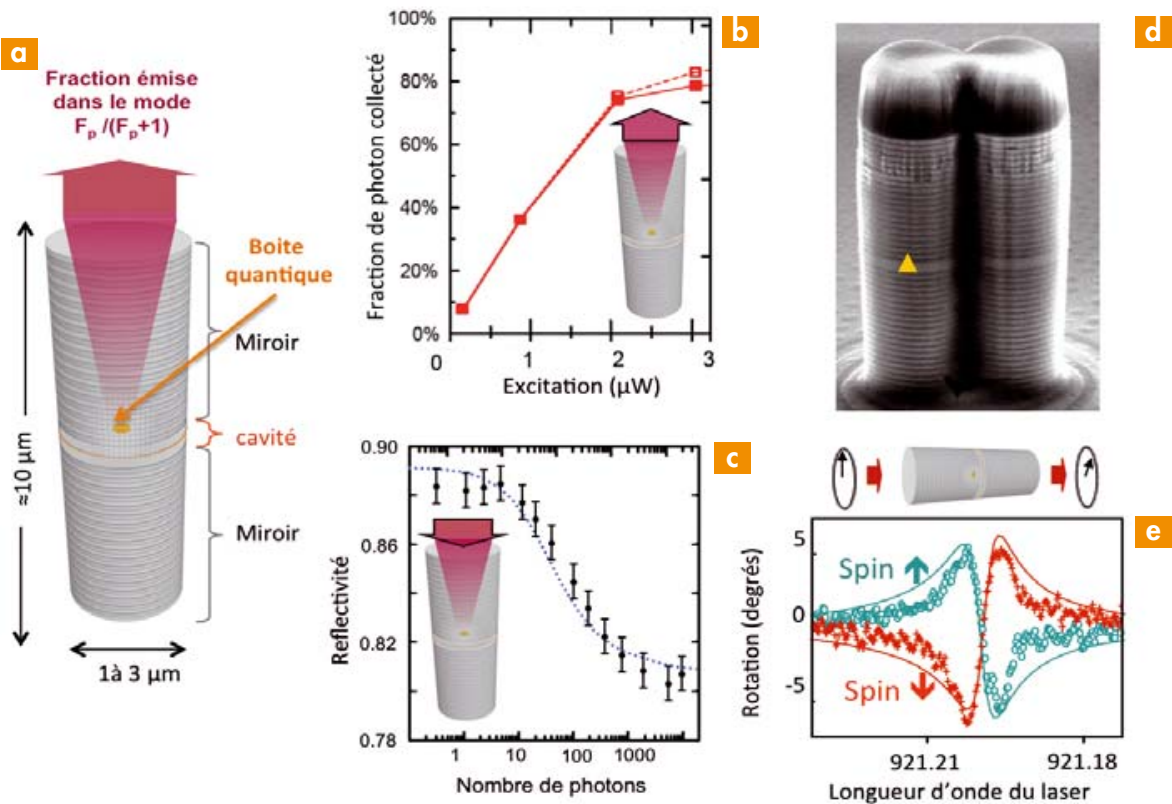

Figure 3. (a) Schéma de principe d'une cavité micropilier et de l'extraction de lumière par accé lération de l'émission spontanée. (b) Probabilité de collecter le photon unique émis par la boîte quantique en fonction de la puissance d'excitation. À saturation, la brillance est de $80 \%$ [8]. (c) Démonstration d'une non-linéarité optique obtenue pour 8 photons seulement en mesurant la réflectivité d'une boîte en cavité [10]. (d) Image obtenue au microscope électronique d'une source ultrabrillante de paires de photons intriqués en polarisation [7]. (e) Une rotation de la polarisation de $\pm 6^{\circ}$ est induite par un spin unique en cavité, suivant que le spin est haut ou bas [1 1]. 
inchangé. Ainsi, la boîte quantique émet son photon unique dans le mode de cavité avec une probabilité $p=F_{\mathrm{p}} /\left(F_{\mathrm{p}}+1\right)$. Avec une accélération de l'émission spontanée modérée, de l'ordre de $F_{\mathrm{p}}=3-5$, cette probabilité atteint près de $75-85 \%$. En choisissant astucieusement la géométrie des cavités, des sources émettant à la demande des photons uniques avec une brillance de $80 \%$ sont maintenant réalisées de façon contrôlée et reproductible [8]. Ces sources sont 20 fois plus brillantes que toute source utilisée aujourd'hui dans le domaine de l'information quantique optique. De plus, le temps d'émission d'un photon unique par une boite quantique est de l'ordre de la centaine de picosecondes, si bien que ces sources peuvent être utilisées à des fréquences allant jusqu'au GHz.

\section{Tout un potentiel à explorer}

De telles sources de photons uniques ont le potentiel d'augmenter de façon critique la complexité de calculs quantiques ainsi que la cadence des communications quantiques. Un des groupes fondateurs du domaine de l'information quantique, celui d'Andrew White à Brisbane en Australie, s'est lancé dans l'aventure il y a deux ans. Avec ce groupe, l'équipe du LPN a déjà mis en place une porte logique quantique avec une source à boîte quantique ultrabrillante [9]. Des expériences d'échantillonnage de bosons sont actuellement en cours de réalisation : le boson sampling est un problème passionnant, et l'un des exemples où un calculateur quantique aurait des capacités supérieures à celles d'un ordinateur classique.

Mais la lithographie optique in situ ne permet pas seulement de fabriquer des sources de photons uniques efficaces. Avec une géométrie de cavité astucieuse, il a aussi été possible de réaliser les sources de paires de photons intriqués en polarisation les plus brillantes à ce jour [7]. De même, s'il est possible de collecter très efficacement les photons uniques émis par une boîte quantique, il est aussi possible, de façon tout à fait symétrique, de s'assurer que tout photon envoyé sur le composant interagisse avec la boîte. Cette propriétéa permis de démontrer des non-linéarités optiques à l'échelle de quelques photons seulement, ouvrant la voie à l'implémentation de portes logiques à l'échelle du photon unique [10]. Et le jeu ne s'arrête pas là, car en insérant un porteur de charge dans la boîte quantique, il est aussi possible de créer un composant qui tourne la polarisation d'un photon de plusieurs degrés suivant que le spin de ce porteur est dans un état haut ou bas [11]. À partir de là, les idées foisonnent. On peut désormais envisager de créer un réseau quantique à l'état solide, où les photons générés par les boîtes quantiques seraient des bits quantiques « volants » faisant le lien entre deux mémoires quantiques constituées de deux spins. Tout cela avec des composants réalisés avec les outils de l'optoélectronique!

\section{Pour en savoir plus}

[1] J.-Y. Marzin, J.-M. Gérard, A. Izraël, D. Barrier, G. Bastard, Phys. Rev. Lett. 73, 716 (1994)

[2] P. Michler, A. Kiraz, C. Becher, W.V. Schoenfeld, P.M. Petroff, Lidong Zhang, E. Hu, A. Imamoglu, Science 290, 2282 (2000)

[3] E.M. Purcell, Phys. Rev. 69, 674 (1946)

[4] A. Badolato, K. Hennessy, M. Atatüre, J. Dreiser, P.M. Petroff, A. Imamoglu, Science 308, 1158 (2005)

[5] A. Dousse, L. Lanco, J. Suffczynski, E. Semenova, A. Miard, A. Lemaître, I. Sagnes, C. Roblin, J. Bloch, P. Senellart, Phys. Rev. Lett. 101, 267404 (2008)

[6] A.K. Nowak, S.L. Portalupi, V. Giesz, O. Gazzano, C. Dal Savio, P.-F. Braun, K. Karrai, C. Arnold, L. Lanco, I. Sagnes, A. Lemaître, P. Senellart, Nature Commun. 5, 3240 (2014)

[7] A. Dousse, J. Suffczynski, A. Beveratos, O. Krebs, A. Lemaître, I. Sagnes, J. Bloch, P. Voisin, P. Senellart, Nature 466, 217 (2010)

[8] O. Gazzano, S. Michaelis de Vasconcellos, C. Arnold, A.K. Nowak, E. Galopin, I. Sagnes, L. Lanco, A. Lemaître, P. Senellart, Nature Commun. 4, 1425 (2013)

[9] O. Gazzano, M.P. Almeida, A.K. Nowak, S.L. Portalupi, A. Lemaître, I. Sagnes, A.G. White, P. Senellart, Phys. Rev. Lett. 110, 250501 (2013)

[10] V. Loo, C. Arnold, O. Gazzano, A. Lemaître, I. Sagnes, O. Krebs, P. Voisin, P. Senellart, L. Lanco, Phys. Rev. Lett. 109, 166806 (2012)

[1 1] C. Arnold, J. Demory, V. Loo, A. Lemaître, I. Sagnes, M. Glazov, O. Krebs, P. Voisin, P. Senellart, L. Lanco, Nature Commun. 6, $6236(2015)$

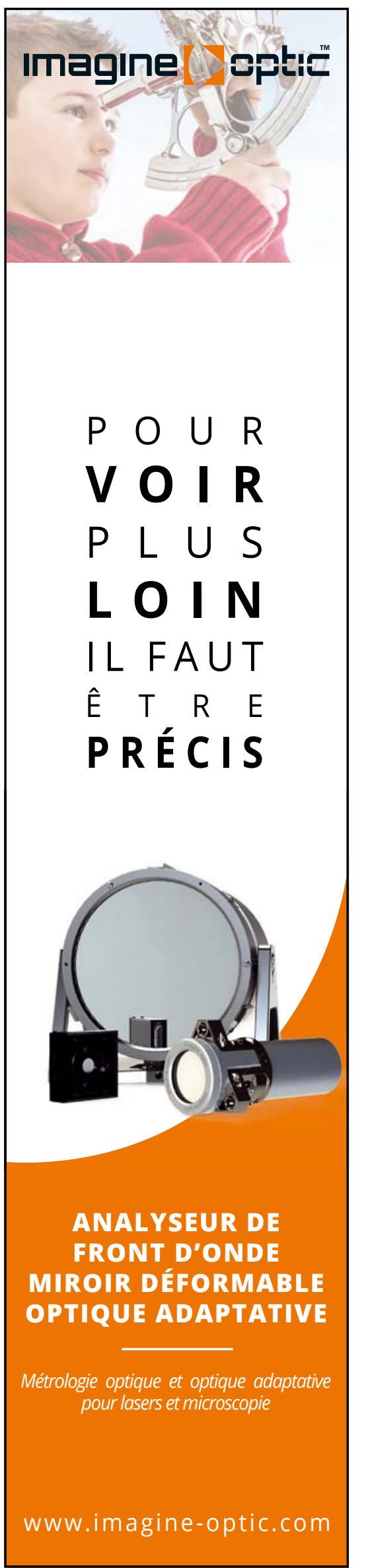


DOMINATIONS

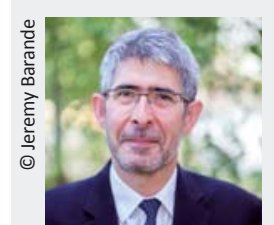

Gilles Bloch, Président de l'université Paris-Saclay

Gilles Bloch est élu Président de I'Université Paris-Saclay par le Conseil d'administration de la ComUE. II succède à Dominique Vernay, qui assurait cette fonction depuis janvier 2015. Gilles Bloch sera aussi Président de la Fondation de Coopération Scientifique Paris-Saclay qui coordonne l'opération campus et le projet Idex. Biophysicien et médecin, ingénieur polytechnicien, Gilles Bloch est Directeur des Sciences du Vivant du CEA depuis septembre 2009.

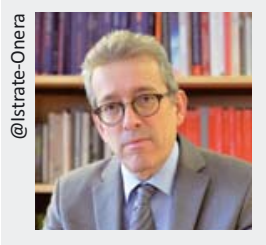

Stéphane Andrieux, Directeur scientifique général de l'ONERA

Stéphane Andrieux vient d'être nommé Directeur scientifique général de I'ONERA. II succède à Emmanuel Rosencher, décédé en mai

2013, et à Pierre Touboul, qui a assuré l'intérim de la fonction jusqu'à son arrivée. Stéphane Andrieux est diplômé de l'Ecole Nationale des Ponts et Chaussées, docteur-ingénieur et habilité à diriger les recherches. Lauréat du Prix Déchelle de l'Académie des Sciences en 2006 et professeur chargé de cours à l'Ecole polytechnique entre 1996 et 2008, il est également professeur à l'Ecole des Ponts Paris Tech.

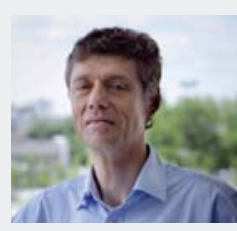

\section{José Garcia rejoint Pyla}

José Garcia a rejoint l'équipe opérationnelle de Pyla, le centre de formation du pôle de compétitivité Route des Lasers. Ingénieur diplômé de l'Ecole supérieure d'optique (aujourd'hui Institut d'optique Graduate School), José Garcia a fondé en 1989 la société Lasoptic ; il poursuivra ses activités d'études, de conseils et de formation au sein du centre de formation Pyla où il renforcera le pôle Sécurité Laser.

\section{PRIX ET DISTINCTIONS}

\section{Giorgio Santarelli}

lauréat du prix EFTF

Le prix 2015 de la conférence européenne du temps et des fréquences (European Frequency and Time Forum) a été remis à Giorgio Santarelli. Ses travaux ont été menés au laboratoire Systèmes de référence temps-espace (SYRTE, Observatoire de Paris/CNRS/UPMC/LNE) ont permis des avancées majeures pour les horloges atomiques micro-ondes et optiques, pour les peignes de fréquences et les liens optiques fibrés. Les résultats qu'il a obtenus notamment dans le domaine des liens optiques fibrés longue distance, en collaboration avec le Laboratoire de physique des lasers (LPL, CNRS / Université Paris 13), ouvrent la voie vers la comparaison d'horloges atomiques de grande précision.

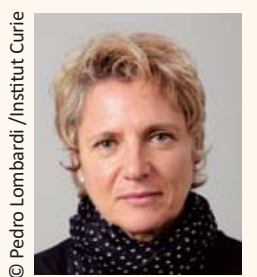

Marie-Paule Teulade-Fichou, médaille d'argent du CNRS 2015

Marie-Paule TeuladeFichou, directeur de recherche CNRS, est spécialiste des acides nucléiques composants de base de la molécule d'ADN, plus particulièrement des G-quadruplexes, structures non-usuelles se formant dans les domaines génomiques riches en guanines, et qui présentent un potentiel thérapeutique dans le traitement du cancer. En 2007, Marie-Paule Teulade-Fichou rejoint l'Institut Curie en tant que directrice adjointe de l'unité de conception, synthèse et vectorisation de biomolécules. Son équipe développe plusieurs familles de molécules distinctes et explore leur potentiel thérapeutique en collaboration avec des biologistes et des cliniciens. Elle est aujourd'hui Directrice de I'unité Chimie, Modélisation et Imagerie pour la Biologie (CMIB) créée sur le site d'Orsay de l'Institut Curie en 2015 , où elle $a$ élargi le champ de ses recherches à la mise au point de sondes fluorescentes ultrabrillantes pour l'imagerie en milieu cellulaire.

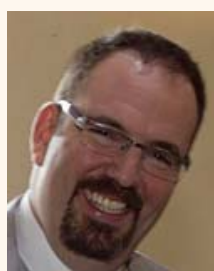

\section{Mathieu Kociak lauréat d'une médaille de la Royal Society of Microscopy \\ Mathieu Kociak a reçu} la médaille Alan Agar de la Royal Society of Microscopy (Grande Bretagne), distinction qui récompense « le fondement d'une unification entre microscopies électroniques et optiques ". Les travaux de son équipe ont joué un rôle moteur pour l'émergence de spectroscopies issues de la microscopie électronique (cathodoluminescence et spectroscopie de pertes d'énergie d'électrons) comme techniques de choix pour la nanooptique. Cela comprend des développements à la fois théoriques et expérimentaux, ainsi qu'un important effort en instrumentation - en particulier la conception et la réalisation d'un système de détection de cathodoluminescence dans un microscope électronique en transmission (STEM).

\section{Philippe Bouyer élu Fellow de l'APS}

Philippe Bouyer a été élu fellow de l'American Physical Society, pour ses travaux sur l'interférométrie atomique avec des atomes ultrafroids et de ses expériences avec des gaz quantiques dégénérés. II a notamment été le collaborateur d'Alain Aspect au laboratoire Charles Fabry à Palaiseau où il a réalisé plusieurs premières, dont la réalisation pour la première fois $d^{\prime}$ une mesure inertielle avec un interféromètre atomique aéroporté, le premier laser à atomes guidés et l'observation de la localisation d'Anderson avec des ondes atomiques. Philippe Bouyer est aussi cofondateur de la société MuQuans qui commercialise les produits issus de son activité de recherche sur l'interférométrie atomique. 